\title{
Angle of repose revisited: When is a heap a cone?
}

\author{
Michael Rackl ${ }^{1, \star}$, Florian E. Grötsch ${ }^{1}$, and Willibald A. Günthner ${ }^{1}$ \\ ${ }^{1}$ Institute for Materials Handling, Material Flow and Logistics \\ Technical University of Munich, Boltzmannstraße 15, 85748 Garching, Germany
}

\begin{abstract}
The angle of repose is a frequently reported value in bulk solids characterisation and its popularity is based on the simple measurement setup. It can be useful to estimate the flowability of a bulk material or to estimate the inner friction coefficient for cohesionless solids. The latter relies on Coulomb material theory, which yields that a bulk solid heap resembles a perfect cone. The aim of this study was to investigate if the cone assumption is valid for three distinct bulk materials. In order to address this question, the angle of repose was measured for each sample and the resulting ideal cone model compared with a respective 3D-scanned surface model of the physical heap. Differences were mapped onto a two-dimensional representation of the ideal cone's lateral surface. The heaps of two of the three investigated bulk solids showed considerable differences with regard to the cone assumption. Discrepancies can be divided into local and global shape deviations. The results show that measuring and reporting the angle of repose should be undertaken with care. Many publications list angle of repose values without reporting the measurement setup or the shape of the heap. However, the latter can vary significantly from Coulomb theory.
\end{abstract}

\section{Introduction}

The angle of repose is a frequently reported value in bulk solids characterisation. Its prevalence is mostly based on the simple and straightforward measurement setup. Furthermore, Coulomb material theory allows for estimation of the bulk solid's inner friction coefficient from the angle of repose. In doing so, the angle of repose measurement relies on the assumption that the concerning bulk solid heap resembles the geometric shape of a perfect cone, which roughly holds true for free-flowing materials. For cohesive or other non-free-flowing bulk solids the question arises, whether the angle of repose is a meaningful characteristic to measure and report. Local effects of cohesion or widely spread variations of particle shapes can result in the physical heaps' shapes to considerably differ from a cone. The aim of this study was to investigate if the cone assumption is valid for lime stone, corn grains and milk powder.

\section{Materials and Methods}

Three-dimensional surface scans of bulk solid heaps were compared against cones based on an ideal Coulomb material.

\subsection{Angle of repose theory}

An ideal bulk solid cone with the angle of repose, $\Phi$, is shown on the left of Figure 1. If one considers an ideal

\footnotetext{
^e-mail: rackl@fml.mw.tum.de,mi.rackl@gmx.de
}
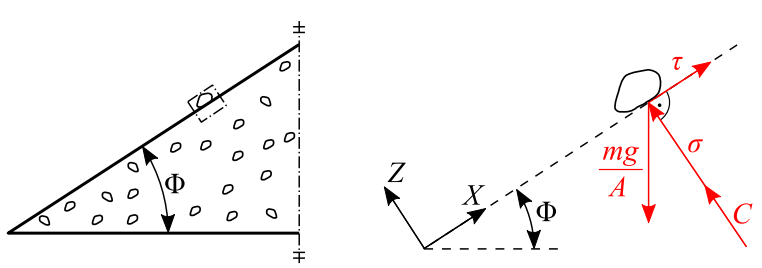

Figure 1. Ideal bulk solid cone (left) and free body diagram of one particle on the lateral surface (right).

Coulomb material [1, p. 55] then the right part of Figure 1 is the free body diagram of a single, tiny particle sitting on other particles of the heap. The equations for the static equilibrium with regard to the axes $X$ and $Z$ are Equations 1 and 2, where $\tau$ is shear stress, $\sigma$ is normal stress, $m$ is the particle mass, $g$ the gravitational acceleration and $A$ the contact surface area between particle and the heap. $C$ is a constant representing cohesional stress between the particle and the surface.

$$
\begin{aligned}
& X: 0=\tau-\frac{m g}{A} \sin \Phi \\
& Z: 0=\sigma+C-\frac{m g}{A} \cos \Phi
\end{aligned}
$$

The combination of both equations yields Equation 3 . After the cohesion constant, $C_{0}=C \tan \Phi$, is introduced and $\tan \Phi$ is replaced by the internal friction coefficient, $\mu$, the Coulomb yield criterion is found in Equation 4 (cf. [1, 
p. 55]); for cohesionless materials: $C_{0} \approx 0$.

$$
\begin{aligned}
& \tau=\sigma \tan \Phi+C \tan \Phi \\
& \tau=\mu \sigma+C_{0}
\end{aligned}
$$

A particle sitting on the lateral surface of a heap will only stay in place if $\tau \geq \mu \sigma+C_{0}$. Otherwise particles are going to start sliding under the influence of gravity until $\tau \geq \mu \sigma+C_{0}$ is satisfied. Hence, for an ideal cohesionless Coulomb material the angle of repose is equal to $\arctan \mu$ and the resulting heap resembles a perfect cone.

\subsection{Experimental investigation of the angle of repose}

The materials investigated in this study were lime stone, corn grains and skimmed milk powder. Graded lime stones with a size range from 20 to $70 \mathrm{~mm}\left(d_{50}=26 \mathrm{~mm}\right)$ were purchased from a local quarry [2]. $d_{50}$ is the mass median diameter as obtained from the particle size distribution. The dried corn grains were obtained from an agricultural wholesale trader [3] and had $d_{50}=6 \mathrm{~mm}$. The investigated skimmed milk powder [4] had a $d_{50}$ of $0.06 \mathrm{~mm}$.

Slump tests were carried out with a cylindrical container $(\varnothing 425 \mathrm{~mm})$ made from rolled mild steel, which was placed on a bottom plate made from the same material. The container was filled to a height of $545 \mathrm{~mm}$ and then lifted up vertically at a constant velocity of $142 \mathrm{~mm} / \mathrm{s}$, such that a heap of the respective bulk solid was formed under the influence of gravity (cf. Figure 2). The angle of repose of the respective heaps was measured according to an FEM standard [5]. In addition to this, the heaps were scanned by means of a KINECT v2 sensor to generate three-dimensional surface meshes. Each measurement was repeated for four heaps $(N=4)$.

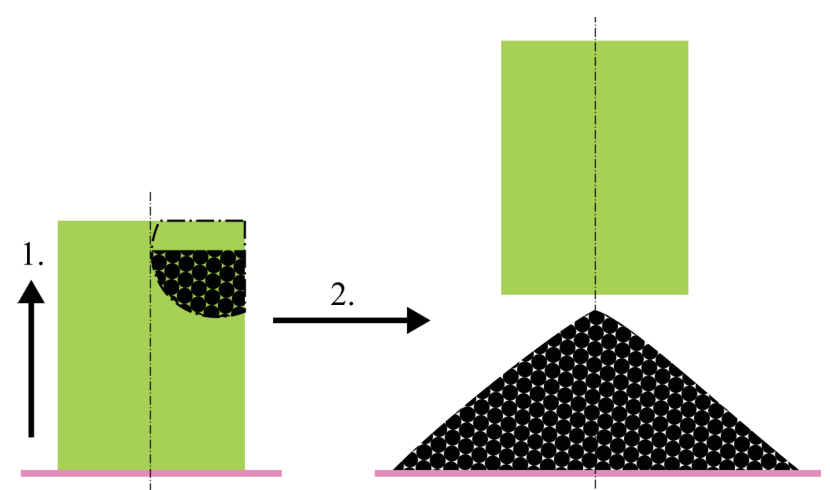

Figure 2. Experimental setup.

Each 3D-scanned surface mesh was imported into Matlab [6] and optimally aligned and positioned with regard to its ideal cone according to FEM. Figure 3 shows an exemplary image of an ideal cone and a 3D-scanned heap surface. The ideal cone was created based on the measured value of the angle of repose and heap height. After the alignment, differences between the surface geometry of the ideal cone and the 3D-scanned surface mesh were determined. Each difference was computed as the distance between a point on the scanned surface mesh and a point on the cone's lateral surface, along a vector perpendicular to the cone's surface [7]. Finally, the differences were mapped onto a two-dimensional developed view of the ideal cone's lateral surface.
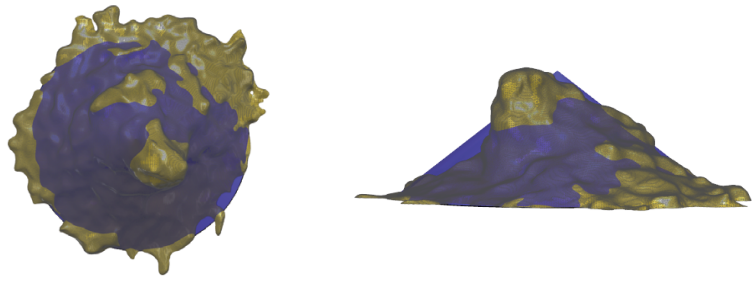

Figure 3. 3D-scanned heap surface (yellow), aligned with regard to its ideal cone counterpart (blue), in a top-town (left) as well as side view (right, not to scale).

\section{Results and Discussion}

Two out of three heap shapes measured by means of threedimensional scans revealed considerable differences with respect to an ideal cone.

\subsection{Angle of repose}

The angle of repose results, measured according to the FEM standard [5], are listed in Table 1. Based on the angle of repose, descriptions of flowability can be assigned to the materials [1, p. 52], whereas lime stone and corn grains would be "very free flowing", while milk powder is considered "free-flowing". These descriptions are of course very rough, since they are exclusively based on the angle of repose.

In addition to this, the ratio between the heap diameter and the particle diameter, $d_{50}$, is listed in Table 1 . The FEM standard suggests that this ratio should exceed 20 for angle of repose measurements.

Table 1. Measured angles of repose with standard deviation and heap diameter to $d_{50}$ particle size ratio. $(N=4)$.

\begin{tabular}{lcc}
\hline Material & Angle of repose $\left(^{\circ}\right)$ & Ratio $(-)$ \\
\hline Lime stone & $26 \pm 1$ & $\sim 38$ \\
\hline Corn grains & $16 \pm 1$ & $\sim 192$ \\
\hline Milk powder & $32 \pm 0$ & $\sim 17,500$ \\
\hline
\end{tabular}

\subsection{Comparison of the angle of repose with the heap surfaces}

Results for the mapped differences between the 3Dscanned heaps and the respective ideal cones for exemplary heaps of each material are shown in the Figures 4, 5 and 6. 
Figure 4 shows good overall agreement in shape, i. e., large areas with small differences. Nonetheless, there are spots, displayed in deep blue and red respectively, where differences are as large as $40 \mathrm{~mm}$. This noise originates from the coarse granularity of the lime stone particles (cf. ratio in Table 1). Since the single particles are rather large compared to the overall heap size, the surface structure of the lime stone heap is rough.

The size ratio of lime stone in this study is considerably larger than the value of 20 which the FEM standards recommends, which brings up questions regarding the significance of measurements with a low heap diameter to particle size ratio. Fluctuations and fluctuation-induced measurement errors of the angle of repose are expected to increase considerably when the size ratio is lower than in this study. The recommended minimum ratio of 20 seems much too low and 38 from this work is on the borderline, at best.

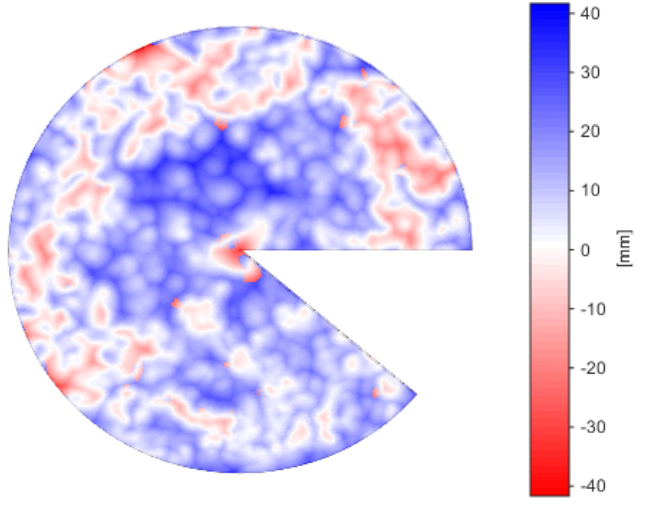

Figure 4. Developed view of the ideal cone with differences to the scanned heap for lime stone.

The developed view for corn grains is depicted in Figure 5. Its surface shows significantly less granularity than that of lime stone and hence appears smoother. Furthermore, the absolute differences between the ideal cone and the scanned heap are considerably smaller. However, a systematically different shape can be observed in a ring around the developed view's centre. From the top to the bottom of the physical heap, i. e. the inside to the outside of the circle sector, the differences increase to a maximum and then decrease until they approximately reach zero again at the bottom of the heap.

Figure 6 displays the result for a milk powder heap. The heap's surface is very smooth due to the tiny particle size of the milk powder. Nonetheless, there are large differences with regard to the ideal cone and the transition between ditches and knobs, i. e. negative and positive differences, is abrupt. In addition to this, the heap is far from rotationally symmetrical.

The causes of the asymmetrical shape of the milk powder heaps are supposedly connected to avalanching effects. When the container was lifted, the powder did not begin

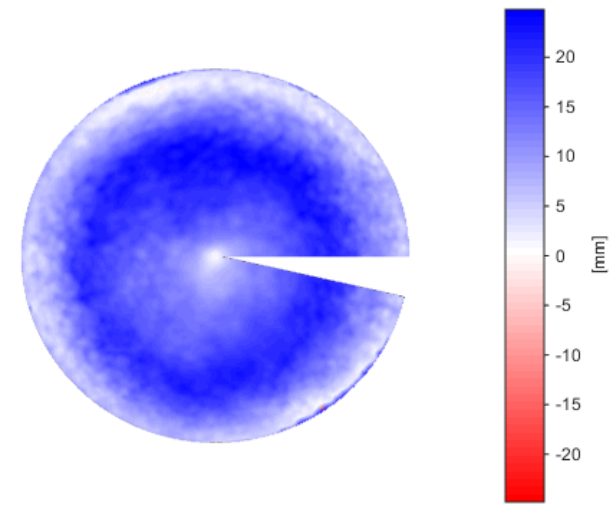

Figure 5. Developed view of the ideal cone with differences to the scanned heap for corn grains.

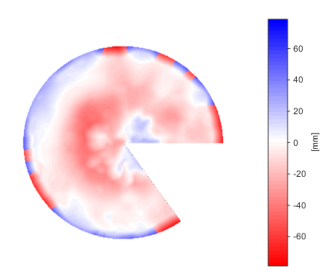

Figure 6. Developed view of the ideal cone with differences to the scanned heap for milk powder.

to flow immediately, but instead kept its cylindrical shape on the first few centimetres, until the cohesive strength was exceeded. Thereafter, larger amounts of milk powder started to pour out of the container intermittently, where each batch generated a small avalanche. Thus, the final shape is assumed to be caused by landslides, which occur when the shear strength and cohesive strength within the milk powder are locally exceeded.

\subsection{Conclusion and outlook}

The main findings with regard to the shape deviations for the three investigated materials are displayed in Figure 7. Lime stone came closest to the global shape of a cone. Yet, its overall shape quality was disturbed by local shape deviations originating from the coarse granularity, i. e. large particle size. The influence of large particle size on the surface smoothness can be seen in the leftmost sketch in Figure 7. The particles form gaps between them and lead to the granular characteristic, where the overall shape fits, yet positive and negative deviations can be observed. The corn grain's heap is similar to a cone and smooth in shape, however the heap has a rounded tip, as shown in the middle of Figure 7. This is in agreement with findings reported in the literature [8]. The heap shape for milk powder is depicted in the rightmost part of Figure 7. It becomes obvious that the angle of repose cannot be directly measured 

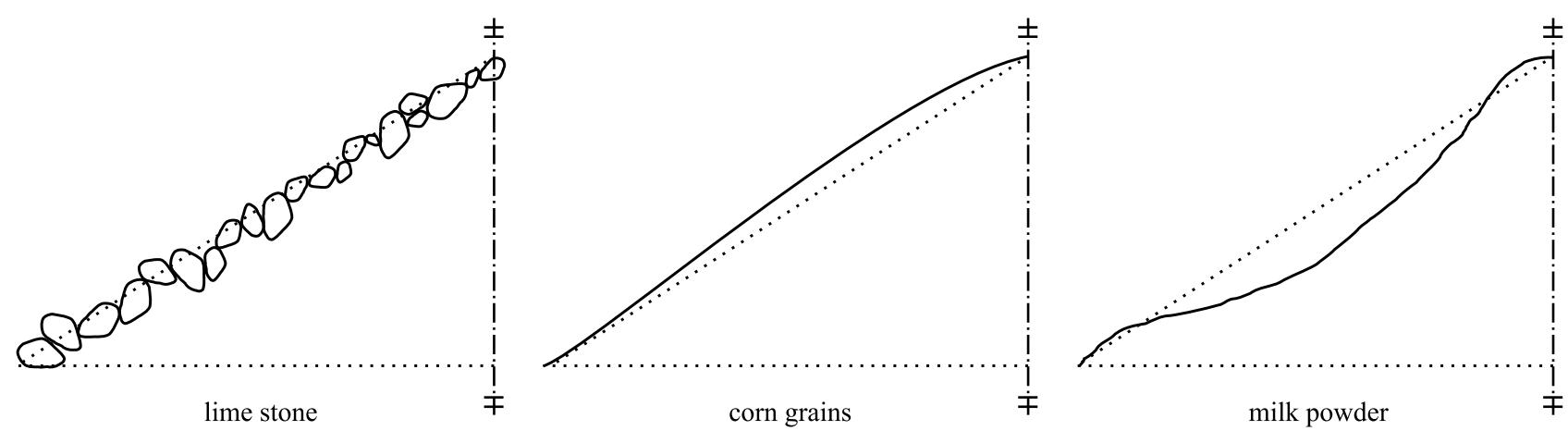

Figure 7. Approximate shape of each of the heaps (solid lines) displayed over the ideal cone (dotted lines).

from a side view, since the inclination changes considerably from the centre to the edge.

The aim of this study was to investigate if the implicit cone assumption of the angle of repose is valid for lime stone, corn grains and milk powder. It can be seen from the scope of the results of this study that this assumption is only valid for lime stone, with the exception of local shape deviations due to its granularity. Corn grains and milk powder show considerable global deviations from the ideal cone shape. Strictly speaking, milk powder is cohesive and hence its heap shape should not be compared with the cone of an ideal cohesionless material. Although cohesive bulk solids do conform to angle of repose theory, the angle of repose is widely reported for such materials in practice [9-11] and can be useful to identify cohesive materials. Corn grains can be considered "free-flowing" according to their angle of repose, however this does not mean that their heap shape resembles a perfect cone. Even though in this study the angle of repose's standard deviation was the smallest for milk powder, its heap shape showed the largest differences from a cone.

Care has to be taken when choosing the dimensions of the angle of repose measurement setup. The results form this work indicate that a heap diameter to particle size ratio of 20 (recommended from the FEM standard) and even 38 (this work) may not be large enough to form heaps which comply with angle of repose theory.

Many publications list values for the angle of repose without reporting the measurement setup or the shape of the heap. The latter however can differ significantly from theoretical considerations, as shown in this study. Future research could focus on three-dimensional scanning of a large variety of bulk materials and quantitative comparison with ideal cones, as well as among each other. These results are going to be helpful in gaining better understanding with regard to heap formation and could provide a base for the link between Coulomb material theory and physical observations of the angle of repose.

\section{Acknowledgement}

This study received funding by the AiF (no. $18371 \mathrm{~N} / 1$ ), within the programme for sponsorship by Industrial Joint Research (IGF) of the German Federal Ministry of Economic Affairs and Energy based on an enactment of the German Parliament.

\section{References}

[1] D. McGlinchey, Characterisation of bulk solids (Blackwell and CRC Press, Oxford and Boca Raton, FL, 2005), ISBN 1-4051-1624-2

[2] Märker Kalk GmbH, Harburg, Germany, Märker lump lime: Technical Data Sheet

[3] BayWa AG, Bockhorn, Germany, Dried corn grains, batch 63, harvest 2014

[4] EUROSÉRUM, Port-sur-Saône, France, Skimmed milk MH PREMIUM - ADPI Extra grade

[5] The European Federation of Materials Handling "FEM", FEM 2.582 (11.1991): General properties of bulk materials and their symbolization

[6] Matlab, version R2014b (The MathWorks Inc, Natick, Massachusetts, 2014)

[7] M. Rusch, Studie zur 3D-gestützten Analyse von Schütthaufen (semester thesis) (16.12.2015), Institute for Materials Handling, Material Flow and Logistics. Technical University of Munich. Germany.

[8] E. Kermani, T. Qiu, T. Li, Int. J. Geomech. 15, 04015004 (2015)

[9] A. Sharma, A.H. Jana, R.S. Chavan, Compr. Rev. Food Sci. F. 11, 518 (2012)

[10] G. Lumay, F. Boschini, K. Traina, S. Bontempi, J.C. Remy, R. Cloots, N. Vandewalle, Powder Technol. 224, 19 (2012)

[11] A. Samadani, A. Kudrolli, Phys. Rev. E 64, 051301 (2001) 